\title{
An efficient ultrasound promoted catalyst-free protocol for the synthesis of chromeno[4,3-b]quinolin-6-ones
}

\author{
J VENKATA PRASAD, J SATYANARAYANA REDDY, N RAVI KUMAR, \\ K ANAND SOLOMON and G GOPIKRISHNA* \\ Sankar Foundation Research Institute, Visakhapatnam 530047, India \\ e-mail: gkrishnagps@gmail.com
}

MS received 31 December 2010; revised 24 May 2011; accepted 30 May 2011

\begin{abstract}
A convenient, catalyst-free protocol for the quantitative synthesis of fused chromeno[4,3b]quinolin-6-ones has been developed by simple one-pot reaction of substituted anilines with 4-chloro-3formylcoumarin using ultrasound irradiation. The protocol offers the advantages of mild reaction conditions, short reaction times and high yields.
\end{abstract}

Keywords. Ultrasound; 4-chloro-3-formylcoumarin; anilines; chromeno-quinoline.

\section{Introduction}

Functionalized quinoline derivatives are integral component of several natural products which are used as antibacterial, ${ }^{1}$ antiasthmatic, ${ }^{2}$ antifungal, ${ }^{3}$ antiinflammatory ${ }^{4}$ and anticancer ${ }^{5}$ agents. Further, they are qualified as valuable starting materials for the synthesis of nano and mesostructures with improved electronic and photonic properties. ${ }^{6}$ The synthesis of quinoline derivatives thus continues to be an active area of heterocyclic chemistry research, and the synthesis of various substituted quinolines has been largely explored. ${ }^{7}$ On the other hand, coumarin derivatives are known to be equally important molecules endowed with wide spectrum of medicinal properties including antibacterial, ${ }^{8}$ antiinflammatory, ${ }^{9}$ antitumor ${ }^{10}$ and anti-HIV ${ }^{11}$ activities. They are also used as perfumes, ${ }^{12}$ dyes ${ }^{13}$ and fluorescent indicators. ${ }^{14}$ Chromeno-quinolines are fused poly heterocyclic systems comprising both coumarin and quinoline motifs which are known to possess interesting biological properties like bacteriostatic activity, ${ }^{15}$ glucocorticoid modulators, ${ }^{16}$ antiinflammatory effects ${ }^{17}$ and selective progesterone receptor modulators. ${ }^{18}$ Thus the structural features and the biological applications prompt intense research by the organic chemists for the development of novel methodologies for their synthesis.

\footnotetext{
${ }^{*}$ For correspondence
}

\section{Experimental}

\subsection{General remarks}

${ }^{1} \mathrm{HNMR}$ spectra were recorded at $400 \mathrm{MHz}$ with a Varian Gemini FT-NMR spectrometer. The chemical shifts are reported in $\delta \mathrm{ppm}$ relative to tetramethylsilane (TMS). The Fourier transform FT-IR spectra were recorded using a Perkin-Elmer 16650 FT-IR spectrometer. The solvents and reagents were used without further purification.

\subsection{Synthesis of chromeno[4,3-b]quinolin-6-one $(3 a-n)$}

A mixture of 4-Chloro-3-formylcoumarin (1) $(1.0 \mathrm{mmol})$ and anilines $\mathbf{2 a}-\mathbf{n}(1.0 \mathrm{mmol})$ in $5 \mathrm{ml}$ of ethanol were subjected to ultrasound irradiation $(33 \mathrm{KHz})$ for $15 \mathrm{~min}$ resulting in precipitation of white solids which were filtered and washed with methanol to yield $\mathbf{3 a}-\mathbf{n}$ in 94-97\% yield.

2.2a 6H-Chromeno[4,3-b]quinolin-6-one (3a): White solid. ${ }^{1} \mathrm{H}$ NMR $\left(400 \mathrm{MHz}, \mathrm{CDCl}_{3}\right): \delta 9.24(\mathrm{~s}, 1 \mathrm{H})$, $8.85(\mathrm{~m}, 1 \mathrm{H}), 8.25(\mathrm{~d}, 1 \mathrm{H}), 8.04(\mathrm{~m}, 1 \mathrm{H}), 7.91(\mathrm{~m}, 1 \mathrm{H})$, $7.62(\mathrm{~m}, 2 \mathrm{H}), 7.49(\mathrm{~m}, 2 \mathrm{H}) ;{ }^{13} \mathrm{C}$ NMR $(400 \mathrm{MHz}$, $\left.\mathrm{CDCl}_{3}\right): 160.3,158.4,152.7,146.7,134.5,132.4$, 128.6, 128.1, 127.0, 126.6, 124.2, 122.5, 119.6, 118.6, 118.7, 114.4; IR (KBr, $\left.v_{\max /} \mathrm{cm}^{-1}\right)$ : 1735, 1732, 2965; EI-MS: $\mathrm{m} / \mathrm{z}=247.06$. 
2.2b 9-Methyl-6H-chromeno[4,3-b]quinolin-6-one (3b): White solid. ${ }^{1} \mathrm{H}$ NMR $\left(400 \mathrm{MHz}, \mathrm{CDCl}_{3}\right): \delta 9.12$ $(\mathrm{s}, 1 \mathrm{H}), 8.77(\mathrm{~m}, 1 \mathrm{H}), 8.13(\mathrm{~d}, 1 \mathrm{H}), 7.76(\mathrm{~s}, 1 \mathrm{H}), 7.74$ $(\mathrm{d}, 1 \mathrm{H}), 7.60(\mathrm{~d}, 1 \mathrm{H}), 7.58(\mathrm{~m}, 1 \mathrm{H}), 7.40(\mathrm{~m}, 1 \mathrm{H}), 2.59$ (s, 3H). ${ }^{13} \mathrm{C}$ NMR $\left(400 \mathrm{MHz}, \mathrm{CDCl}_{3}\right): 161.4,152.5$, 149.7, 148.8, 139.9, 137.5, 135.7, 131.9, 129.2, 127.8, 127.3, 125.1, 124.8, 119.7, 117.3, 115.7, 21.5; IR (KBr, $\left.v_{\max /} \mathrm{cm}^{-1}\right): 1735,1734,2923,1188$; EI-MS: $\mathrm{m} / \mathrm{z}=$ 261.27

2.2c 9-Chloro-6H-chromeno[4,3-b]quinolin-6-one (3c): White solid. ${ }^{1} \mathrm{H}$ NMR $\left(400 \mathrm{MHz}, \mathrm{CDCl}_{3}\right): \delta 9.15$ $(\mathrm{s}, 1 \mathrm{H}), 8.77(\mathrm{~m}, 1 \mathrm{H}), 8.19(\mathrm{~d}, 1 \mathrm{H}), 8.01(\mathrm{~d}, 1 \mathrm{H}), 7.86$ $(\mathrm{m}, 1 \mathrm{H}), 7.61(\mathrm{~m}, 1 \mathrm{H}), 7.44(\mathrm{~m}, 1 \mathrm{H}), 7.26(\mathrm{~s}, 1 \mathrm{H})$; ${ }^{13} \mathrm{C}$ NMR $\left(400 \mathrm{MHz}, \mathrm{CDCl}_{3}\right): 160.9,152.8,149.9$, 149.5, 139.9, 134.3, 133.4, 132.6, 131.2, 127.8, 127.7, 125.3, 125.1, 119.4, 117.5, 116.6; IR (KBr, $\left.v_{\max /} \mathrm{cm}^{-1}\right)$ : 3061, 1744, 1176; EI-MS: m/z = 281.44

2.2d 9-Nitro-6H-chromeno[4,3-b]quinolin-6-one (3d): Yellow solid. ${ }^{1} \mathrm{H}$ NMR $\left(400 \mathrm{MHz}, \mathrm{CDCl}_{3}\right): \delta 9.18$ (s, 1H), $8.73(\mathrm{~m}, 1 \mathrm{H}), 8.16(\mathrm{~d}, 1 \mathrm{H}), 8.01(\mathrm{~d}, 1 \mathrm{H}), 7.82$ $(\mathrm{m}, 1 \mathrm{H}), 7.60(\mathrm{~m}, 1 \mathrm{H}), 7.44(\mathrm{~m}, 1 \mathrm{H}), 7.24(\mathrm{~s}, 1 \mathrm{H})$; ${ }^{13} \mathrm{C}$ NMR $\left(400 \mathrm{MHz}, \mathrm{CDCl}_{3}\right): 159.8,155.6,152.2$, 143.4, 135.7, 130.6, 126.9, 126.7, 126.3, 125.2, 125.1, 120.4, 118.9, 117.9, 117.7, 112.4; IR (KBr, $\left.v_{\max /} \mathrm{cm}^{-1}\right)$ : 1735, 1743, 1179; EI-MS: m/z = 292.25

2.2e 9-Bromo-6H-chromeno[4,3-b]quinolin-6-one (3e): White solid. ${ }^{1} \mathrm{H}$ NMR $\left(400 \mathrm{MHz}, \mathrm{CDCl}_{3}\right): \delta 9.20$ (s, 1H), $8.76(\mathrm{~m}, 1 \mathrm{H}), 8.45(\mathrm{~s}, 1 \mathrm{H}), 7.89(\mathrm{~d}, 1 \mathrm{H}), 7.73$ $(\mathrm{m}, 1 \mathrm{H}), 7.63(\mathrm{~m}, 1 \mathrm{H}), 7.45(\mathrm{~m}, 1 \mathrm{H}), 7.26(\mathrm{~s}, 1 \mathrm{H})$; ${ }^{13} \mathrm{C}$ NMR $\left(400 \mathrm{MHz}, \mathrm{CDCl}_{3}\right): 162.5,159.4,150.3$, 146.2, 133.3, 132.3, 130.1, 128.1, 127.4, 126.9, 125.5, 125.1, 122.3, 121.3, 118.8, 113.2; IR (KBr, $\left.v_{\max } / \mathrm{c}^{-1}\right)$ : 1737, 1734, 1174; EI-MS: m/z = 326.14.

2.2f 9-Fluoro-6H-chromeno[4,3-b]quinolin-6-one (3f): White solid. ${ }^{1} \mathrm{H}$ NMR $\left(400 \mathrm{MHz}, \mathrm{CDCl}_{3}\right): \delta 9.25$ $(\mathrm{s}, 1 \mathrm{H}), 8.77(\mathrm{~m}, 1 \mathrm{H}), 8.26(\mathrm{~m}, 1 \mathrm{H}), 8.27(\mathrm{~m}, 1 \mathrm{H}), 7.67$ $(\mathrm{m}, 1 \mathrm{H}), 7.58(\mathrm{~m}, 1 \mathrm{H}), 7.45(\mathrm{~m}, 1 \mathrm{H}), 7.26(\mathrm{~s}, 1 \mathrm{H})$; ${ }^{13} \mathrm{C}$ NMR $\left(400 \mathrm{MHz}, \mathrm{CDCl}_{3}\right): 162.6,160.1,158.7$, 154.4, 145.3, 136.6, 132.9, 127.1, 126.4, 122.9, 122.4, 118.2, 116.9, 116.1, 115.9, 113.9; IR (KBr, $\left.v_{\max /} \mathrm{cm}^{-1}\right)$ : 1735, 1740, 1218. EI-MS: $\mathrm{m} / \mathrm{z}=265.4$

$2.2 \mathrm{~g}$ 10-Bromo-6H-chromeno[4,3-b]quinolin-6-one (3g): White solid. ${ }^{1} \mathrm{H}$ NMR $\left(400 \mathrm{MHz}, \mathrm{CDCl}_{3}\right): \delta 9.17$ (s, 1H), $8.75(\mathrm{~m}, 1 \mathrm{H}), 8.46(\mathrm{~s}, 1 \mathrm{H}), 7.90(\mathrm{~d}, 1 \mathrm{H}), 7.73$ $(\mathrm{m}, 1 \mathrm{H}), 7.63(\mathrm{~m}, 1 \mathrm{H}), 7.45(\mathrm{~m}, 1 \mathrm{H}), 7.24(\mathrm{~s}, 1 \mathrm{H})$; ${ }^{13} \mathrm{C}$ NMR $\left(400 \mathrm{MHz}, \mathrm{CDCl}_{3}\right): 161.3,158.4,154.3$, 141.1, 131.6, 130.7, 129.1, 126.9, 126.5, 124.9, 122.3, 123.1, 122.3, 119.8, 117.8, 113.7; IR (KBr, $\left.v_{\max } / \mathrm{c}^{-1}\right)$ : 1735, 1737, 1172; EI-MS: $\mathrm{m} / \mathrm{z}=326.17$.

2.2h 9,10-Difluoro-6H-chromeno[4,3-b]quinolin-6one $(3 \boldsymbol{h})$ : White solid. ${ }^{1} \mathrm{H}$ NMR $\left(400 \mathrm{MHz}, \mathrm{CDCl}_{3}\right)$ : $\delta 9.46(\mathrm{~s}, 1 \mathrm{H}), 8.63(\mathrm{~m}, 1 \mathrm{H}), 8.25(\mathrm{~m}, 1 \mathrm{H}), 7.89(\mathrm{~m}, 2 \mathrm{H})$, $7.70(\mathrm{~m}, 1 \mathrm{H}), 7.52(\mathrm{~m}, 1 \mathrm{H}) ;{ }^{13} \mathrm{C}$ NMR $(400 \mathrm{MHz}$, $\left.\mathrm{CDCl}_{3}\right): 162.7,158.2,155.3,152.8,148.4,134.2$, $128.2,125.5,124.2,120.1,119.2,118.7,118.2,116.5$, 115.4, 115.7; IR (KBr, $\left.v_{\max /} \mathrm{cm}^{-1}\right): 1735,1739,1176$; EI-MS: $\mathrm{m} / \mathrm{z}=283.14$.

$2.2 \mathrm{i}$ 10,11-Difluoro-6H-chromeno[4,3-b]quinolin-6one (3i): White solid. ${ }^{1} \mathrm{H}$ NMR $\left(400 \mathrm{MHz}, \mathrm{CDCl}_{3}\right)$ : $\delta 9.47(\mathrm{~s}, 1 \mathrm{H}), 8.65(\mathrm{~m}, 1 \mathrm{H}), 8.28(\mathrm{~m}, 1 \mathrm{H}), 7.88(\mathrm{~m}, 2 \mathrm{H})$, $7.75(\mathrm{~m}, 1 \mathrm{H}), 7.53(\mathrm{~m}, 1 \mathrm{H}) .{ }^{13} \mathrm{C}$ NMR $(400 \mathrm{MHz}$, $\left.\mathrm{CDCl}_{3}\right)$ : 161.7, 156.2, 153.2, 151.3, 145.3, 136.7, 131.8, 127.4, 127.1, 126.4, 125.6, 124.4, 121.5, 120.3, 119.3, 118.1; IR (KBr, $\left.v_{\max /} \mathrm{cm}^{-1}\right): 3046,1734,1737$, 1178; EI-MS: $\mathrm{m} / \mathrm{z}=283.24$.

2.2j 10-Fluoro-6H-chromeno[4,3-b]quinolin-6-one (3j): White solid. ${ }^{1} \mathrm{H}$ NMR $\left(400 \mathrm{MHz}, \mathrm{CDCl}_{3}\right): \delta 9.21$ $(\mathrm{s}, 1 \mathrm{H}), 8.77(\mathrm{~m}, 1 \mathrm{H}), 8.05(\mathrm{~m}, 1 \mathrm{H}), 7.85(\mathrm{~m}, 1 \mathrm{H})$, $7.62(\mathrm{~m}, 1 \mathrm{H}), 7.42(\mathrm{~m}, 1 \mathrm{H}), 7.39(\mathrm{~d}, 1 \mathrm{H}), 7.26(\mathrm{~s}, 1 \mathrm{H})$; ${ }^{13} \mathrm{C}$ NMR $\left(400 \mathrm{MHz}, \mathrm{CDCl}_{3}\right): 166.9,161.1,152.6$, $150.7,140.9,132.7,131.9,125.4,125.1,119.4,118.7$,

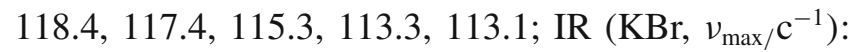
3070, 1739, 1743, 1181; EI-MS: $\mathrm{m} / \mathrm{z}=265.05$.

2.2k 9-Methoxy-6H-chromeno[4,3-b]quinolin-6-one (3k): White solid. ${ }^{1} \mathrm{H}$ NMR $\left(400 \mathrm{MHz}, \mathrm{CDCl}_{3}\right): \delta 9.10$ $(\mathrm{s}, 1 \mathrm{H}), 8.75(\mathrm{~m}, 1 \mathrm{H}), 8.15(\mathrm{~d}, 1 \mathrm{H}), 7.56(\mathrm{~m}, 2 \mathrm{H})$, $7.41(\mathrm{~m}, 2 \mathrm{H}), 7.26(\mathrm{~s}, 1 \mathrm{H}), 3.99(\mathrm{~s}, 3 \mathrm{H}) ;{ }^{13} \mathrm{C} \mathrm{NMR}$ $\left(400 \mathrm{MHz}, \mathrm{CDCl}_{3}\right): 161.5,158.4,152.4,147.7,147.5$, $138.8,132.2,131.7,131.1,128.5,126.9,124.8,119.8$, 117.3, 115.9, 105.6, 55.7; IR (KBr, $\left.v_{\max /} \mathrm{cm}^{-1}\right): 2990$, 1735, 1737, 1237; EI-MS: m/z = 277.07.

2.21 10-Methoxy-6H-chromeno[4,3-b]quinolin-6-one (3l): White solid. ${ }^{1} \mathrm{H}$ NMR $\left(400 \mathrm{MHz}, \mathrm{CDCl}_{3}\right): \delta 9.20$ $(\mathrm{s}, 1 \mathrm{H}), 8.86(\mathrm{~m}, 1 \mathrm{H}), 7.39-7.62(\mathrm{~m}, 5 \mathrm{H}), 7.26(\mathrm{~s}$, 1H), $3.99(\mathrm{~s}, 3 \mathrm{H}) ;{ }^{13} \mathrm{C}$ NMR $\left(400 \mathrm{MHz}, \mathrm{CDCl}_{3}\right): 162.7$, 159.4, 153.4, 148.7, 147.4, 138.4, 132.7, 130.7, 129.1, $128.2,126.4,123.5,118.9,117.7,116.1,104.6,54.6$; IR $\left(\mathrm{KBr}, v_{\max } / \mathrm{cm}^{-1}\right): 3063,1734,1735,1181$; EI-MS: $\mathrm{m} / \mathrm{z}=277.07$. 
$2.2 \mathrm{~m}$ 10-Methyl-6H-chromeno[4,3-b]quinolin-6-one (3m): White solid. ${ }^{1} \mathrm{H}$ NMR $\left(400 \mathrm{MHz}, \mathrm{CDCl}_{3}\right)$ : $\delta 9.14(\mathrm{~s}, 1 \mathrm{H}), 8.77(\mathrm{~m}, 1 \mathrm{H}), 8.10(\mathrm{~d}, 1 \mathrm{H}), 7.72$ (s, 1H), $7.70(\mathrm{~d}, 1 \mathrm{H}), 7.60(\mathrm{~d}, 1 \mathrm{H}), 7.57(\mathrm{~m}, 1 \mathrm{H}), 7.40$ $(\mathrm{m}, 1 \mathrm{H}), 2.56(\mathrm{~s}, 3 \mathrm{H}) ;{ }^{13} \mathrm{C}$ NMR $\left(400 \mathrm{MHz}, \mathrm{CDCl}_{3}\right)$ : 161.3, 152.6, 151.2, 149.5, 144.4, 140.3, 132.1, 129.7, $128.9,128.4,125.2,124.8,119.7,117.2,114.9,22.2$; IR $\left(\mathrm{KBr}, v_{\max } / \mathrm{cm}^{-1}\right): 1735,1737,2923,1178$; EI-MS: $\mathrm{m} / \mathrm{z}=261.26$.

2.2n 10-Methoxy-6H-chromeno[4,3-b]quinolin-6-one $(3 n)$ : White solid. ${ }^{1} \mathrm{H}$ NMR $\left(400 \mathrm{MHz}, \mathrm{CDCl}_{3}\right)$ : $\delta 9.65(\mathrm{~s}, 1 \mathrm{H}), 8.75(\mathrm{~m}, 1 \mathrm{H}), 7.89(\mathrm{~d}, 1 \mathrm{H}), 7.81(\mathrm{~d}, 1 \mathrm{H})$, 7.60-7.30 (m, 3H), $7.2(\mathrm{~m}, 1 \mathrm{H}), 4.05(\mathrm{~s}, 3 \mathrm{H}) ;{ }^{13} \mathrm{C}$ NMR $\left(400 \mathrm{MHz}, \mathrm{CDCl}_{3}\right): 161.8,159.4,153.4,148.7,147.4$, 138.4, 132.7, 130.7, 129.1, 128.2, 126.4, 123.5, 118.9,

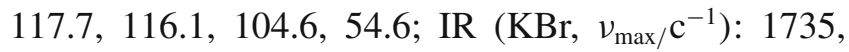
1737, 1178; EI-MS: $\mathrm{m} / \mathrm{z}=277.27$.

\section{Results and discussions}

Chromeno[4,3-b]quinolin-6-one derivatives (3a-n) are important class of chromeno-quinolines and the most conventional approach for their synthesis involves the reaction of 4-hydroxycoumarins with anilines and paraformaldehyde at $220-240^{\circ} \mathrm{C}$ under vacuum. ${ }^{19}$ Tabakovic et al. have reported their synthesis starting from 4-hydroxycoumarin under Vilsmeier-Haack conditions. ${ }^{20}$ Asherson et al. have reported their synthesis from 3-((dimethylamino)methyl)-4-hydroxy- $2 \mathrm{H}$ chromen-2-one and anilines but with lower yields. ${ }^{21}$ Haber et al. have demonstrated stoichiometric $\mathrm{AlCl}_{3}$ catalysed synthesis of the compounds by using 4-chloro-3-formylcoumarin and aniline in refluxing tetrahydrofuran. ${ }^{22}$ Bandyopadhyay et al. ${ }^{23}$ and $\mathrm{Wu}$ et al. ${ }^{24}$ have used 4-oxo-4H-chromene-3-carbaldehyde and 4-chloro-3- formylcoumarin respectively for the synthesis of the chromeno[4,3-b]quinolin-6-ones. All the above methods require either Lewis acid catalysts or high temperatures for their synthesis.
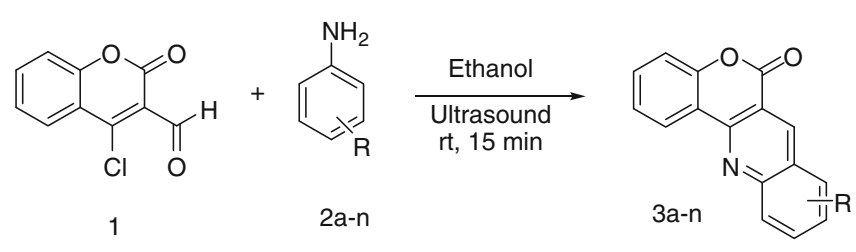

Scheme 1. Synthesis of fused chromeno-quinolines.

Recently, ultrasonication methods have gained wide applicability in several organic synthetic protocols where the reaction conditions have been either modified and/or improved using the ultrasound irradiation. ${ }^{25}$ We have earlier reported L-proline catalysed three component asymmetric Mannich reaction where ultrasonication conditions have efficiently enhanced the rate of the reaction along with improvement in the yields and enantioselectivities. ${ }^{26}$ In view of the enormous potential of chromeno[4,3-b]quinolin-6-ones for various biological applications, we have envisaged an operationally benign catalyst-free protocol for their synthesis under ultrasound irradiation (scheme 1).

First, 4-chloro-3-formylcoumarin and pmethylaniline were subjected to ultrasound irradiation in various solvents. As shown in table 1 (entries 1-6), ultrasonication promotes the formation of the fused chromeno-quinolines $\mathbf{3 b}$ in different solvents with varying reaction rates and yields. The results show that choice of solvent has profound effect on both the rates of reaction and yields. While the reaction did not proceed in water, solvents like dichloromethane and chloroform gave comparatively lower yields $(\sim 30 \%)$ after $30 \mathrm{~min}$ of ultrasound irradiation. Acetonitrile afforded $60 \%$ while methanol afforded $85 \%$ of the required product in 20 and 15 minutes respectively. Ethanol proved to be the solvent of choice which afforded $95 \%$ of the required product as a white precipitate in $15 \mathrm{~min}$ without the requirement for column purification.

The optimized reaction conditions were further extended to the reaction of various aniline substrates possessing diverse electronic features making the protocol rather general and the results are listed in table 2. For example, the electron-rich anilines having $p$-Me, $p$-OMe, $o$-OMe, $m$-Me, $m$-OMe functional groups afforded the respective products in 15 minutes with yield between 94 and 97\% (entries 2, 11-14, table 2). Similarly, the electron withdrawing $p$-nitroaniline afforded the required product in $96 \%$ yield (entry 4 ,

Table 1. Effect of solvents for the synthesis of coumarinoquinolines from 4-chloro-3-formylcoumarin and aniline. ${ }^{\mathrm{a}}$

\begin{tabular}{llcc}
\hline Entry & Solvent & Time (min) & Yield $^{\mathrm{b}}(\%)$ \\
\hline 1 & $\mathrm{C}_{2} \mathrm{H}_{5} \mathrm{OH}$ & 15 & 95 \\
2 & $\mathrm{CH}_{3} \mathrm{OH}$ & 15 & 85 \\
3 & $\mathrm{CH}_{3} \mathrm{CN}$ & 20 & 60 \\
4 & $\mathrm{CH}_{2} \mathrm{CL}_{2}$ & 30 & 30 \\
5 & $\mathrm{CHCl}_{3}$ & 30 & 30 \\
6 & Water & 45 & $\mathrm{NR}^{\mathrm{c}}$
\end{tabular}

${ }^{\mathrm{a}}$ All the reactions were carried out using $\mathbf{1}(1.0 \mathrm{mmol}), 2 \mathbf{b}$ $(1.0 \mathrm{mmol})$ at room temperature under ultrasound conditions, ${ }^{\mathrm{b}}$ determined by ${ }^{1} \mathrm{H}$ NMR.

${ }^{\mathrm{c}}$ No reaction. 
Table 2. Synthesis of chromeno[4,3-b]quinolin-6-ones.

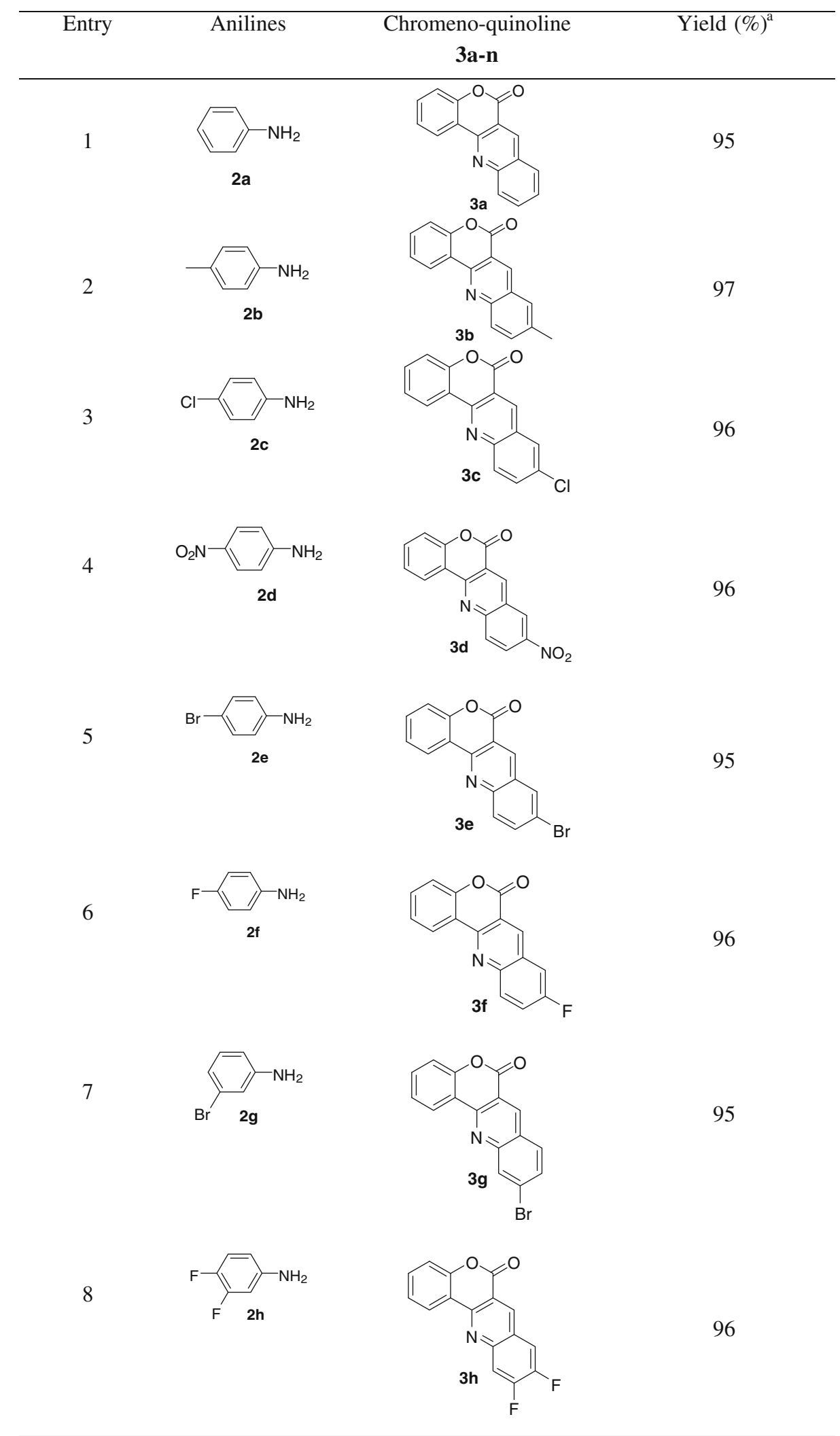

table 2). Anilines having various mono- and di- ones within 15 minutes under ultrasonication conditions substituted halogens also gave satisfactory yields (94- (entries 3, 5-10, table 2). All the products were charac96\%) for the respective chromeno[4,3-b]quinolin-6- terized by ${ }^{1} \mathrm{HNMR}$, IR, and EI-Mass analysis and were 
Table 2. (continued).

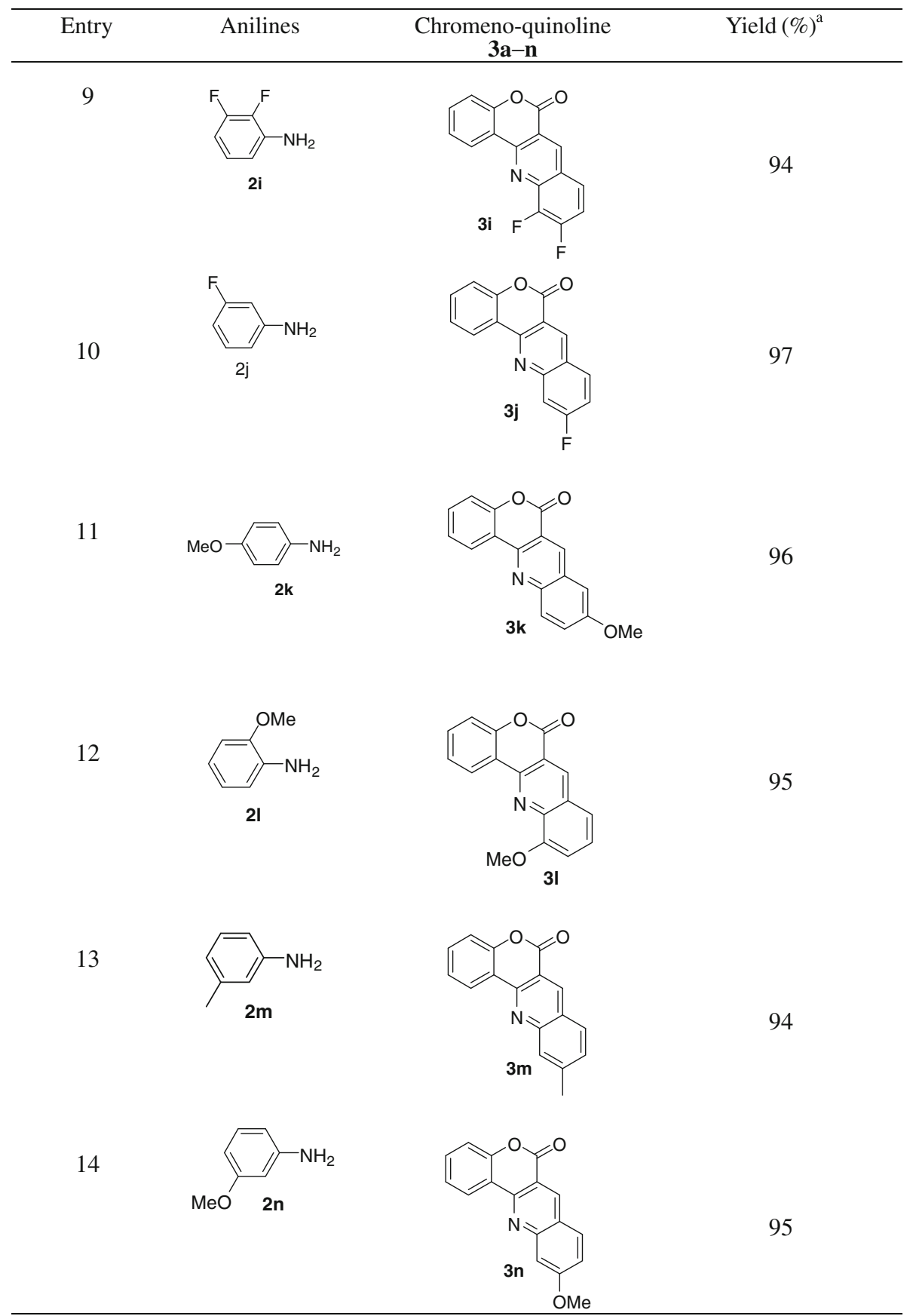

${ }^{a}$ Yields refer to the precipitated products.

found to be in good agreement with those reported in the literature. ${ }^{22}$

A plausible mechanism for the synthesis of $\mathbf{3 a} \mathbf{a} \mathbf{3 n}$ is proposed in scheme 2 . The first step of the reaction involves nucleophilic attack of aniline on $\mathbf{1}$ resulting in the $N$-alkylation intermediate $\mathbf{I}$. The second step involves the activation of the aldehyde group by the in situ generated $\mathrm{HCl}$, which acts as a protic acid promoting the cyclization of the aromatic ring to generate intermediate III. Elimination of the water molecule in the final step affords fused quinoline products 3a-3n. The ultrasound energy in the present protocol efficiently causes acoustic cavitation, which results in extreme temperatures and pressures on microsecond time scale causing dramatic influence on the rates of the reaction. ${ }^{25 \mathrm{~d}}$ 


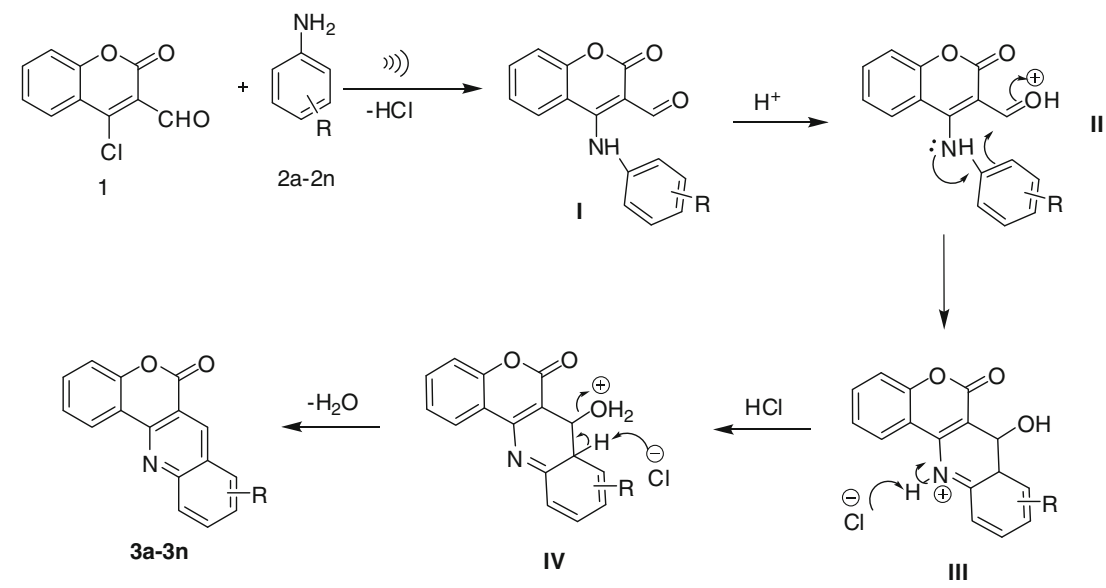

Scheme 2. Plausible mechanism for the synthesis of chromeno[4, 3-b]quinolin-6-ones.

\section{Conclusion}

In conclusion, we have reported an efficient, fast, catalyst-free ultrasonication methodology for the synthesis of fused chromeno[4,3-b]quinolin-6-one derivatives. The present methodology offers the advantages of mild reaction conditions, short reaction times; high yields and avoids the requirement of Lewis acid catalysts. Further, the protocol does not require column chromatography purification.

\section{Acknowledgements}

The authors thank Mr. A Sankar Rao and Mrs. A Yesodha, Founder Trustees of Sankar Foundation, for the kind support and encouragement.

\section{References}

1. Nasveld P, Kitchener S and Trans R 2005 Soc. Trop. Med. Hyg. 992

2. Leatham P A, Bird H A, Wright V, Seymour D, Gordon A 1983 Eur. J. Rheumatol. Inflamm. 6209

3. Denny W A, Wilson W R, Ware D C, Atwell G J, Milbank J B and Stevenson R J 2006 U. S Patent 7064117

4. Mahamoud A, Chevalier J, Davin-Regli A, Barbe J and Pages J M 2006 Curr. Drug Targets. 7843

5. Muruganantham N, Sivakumar R, Anbalagan N, Gunasekaran V and Leonard J T Biol. 2004 Pharm. Bull. 271683

6. Maguire M P, Sheets K R, McVety K, Spada A P and Zilberstein A 1994 J. Med. Chem. 372129

7. For a recent reviews: (a) Gilchrist T L $2001 \mathrm{~J}$. Chem. Soc., Perkin Trans. 1 2491; (b) Kouznetsov V V, Mendez L Y and Gomez C M 2005 Curr. Org. Chem. 9 141; (c) Chambers R D, Holling D, Sandford G, Puschmann $\mathrm{H}$ and Howard J A K 2002 J. Fluorine Chem. 117 99; (d) Chambers R D, Holling D, Sandford G,
Puschmann H and Howard J A K 2004 J. Fluorine Chem. 125 661; (e) Bonnet V, Mongin F, Trecourt F, Queguiner G and Knochel P 2002 Tetrahedron. 58 4429

8. Al-Haiza M A, Mostafa M S and El-Kady M Y 2003 Molecules. 8 275; (b) Musiciki B, Periers A M, Laurin P, Ferroud D, Benedetti Y, Lachaud S, Chatreaux F, Haesslein J L, LLtis A, Pierre C, Khider J, Tessol N, Airault M, Demassey J, Dupuis-Hamelin C, Lassaigne P, Bonnefoy A, Vicat P and Klich M 2000 Bioorg. Med. Chem. Lett. 1016959

9. Fylaktakidou K C, Hadipavlou-Litina D J, Litinas K E and Nicolaides D N 2004 Curr. Pharm. Des. 103813

10. Harvey R G, Cortex C, Ananthanarayan T P and Schmolka S 1988 J. Org. Chem. 533936

11. Kostova I, Raleva S, Genova P and Argirova R 2006 Bioinorg. Chem. Appl. 68274

12. Clark G S 1995 Perfum. Flavor. 2023

13. Sekar N. Colourage. 5055

14. Brun M P, Bischoff L and Garbay C 2004 Angew. Chem., Int. Ed. 433432

15. Liu Y and Ding Y 1995 Ниахие Yanjiu Yu Yingyong. 7 430

16. Elmore S W, Pratt J K, Coghlan M J, Mao Y, Green B E, Anderson D, Stashko M A, Lin C W, Falls D, Nakane M, Miller L, Tyree C M, Miner J N and Lane B 2004 Bioorg. Med. Chem. Lett. 14 1721; (b) Ku Y Y, Grieme T, Raje P, Sharma P, Morton H E, Rozema M and King S A 2003 J. Org. Chem. 683238

17. Kym P R, Kort M E, Coghlan M J, Moore J L, Tang R and Ratajczyk J D 2003 J. Med. Chem. 461016

18. Zhi L, Tegley C M, Pio B, Edward J P, Motamedi M, Jones T D, Marschke K B, Mais D E, Risek B and Schrader W T 2003 J. Med. Chem. 464104

19. Thang D C, Weisberger E K, Mabille P and Buu-Hoi N P 1967 J. Chem. Soc. C. 8 665; (b) Buu-Hoi N P 1967 J. Chem. Soc. C. 3 213; (c) Buu-Hoi N P, Jacquignon P and Mangane M 1971 J. Chem. Soc. C. 10 1991; (d) Tabakovic K, Tabakovic I, Trkovnik M, Juric A and Trinajstic N 1980 J. Heterocycl.Chem. 17801

20. Tabaković K, Tabaković I, Ajdini N and Leci O 1987 Synthesis. 3308 
21. Asherson J L, Bilgic O and Young D W 1980 J. Chem. Soc., Perkin Trans. 1522

22. Heber D 1987 Archiv der Pharmazie. 7595

23. Bandyopadhyay C, Sur K R, Patra R and Sen A 2000 Tetrahedron. 563583

24. Wu J and Wang X 2006 Org. Biomol. Chem. 41348

25. Ley S V, Low C M R 1989 Ultrasound in Synthesis. Series: Reactivity and Structure. New York: Springer; (b) Suslick K S 1988 Ultrasound: Its chemical, physical, and biological effects. New York: VCH; (c) Mason
T J and Phillip L J 1989 Endeavour 13 123; (d) Soheila S, Jean-Marc L and Micheline D 2010 Chem. Natural Comp. 46 75; (e) Muravyova E A, Desenko S M, Musatov V I, Knyazeva I V, Shishkina S V, Shishkin O V and Chebanov V A 2007 J. Comb. Chem. 9 797; (f) Mason T J, Lorimer J P 2002 Applied Sonochemistry. Wiley-VCH, Germany

26. Kantam M L, Rajasekhar Ch V, Gopikrishna G, Rajender R K and Choudary B M 2006 Tetrahedron Lett. 475965 